\title{
Vagal denervation and reinnervation after ablation of ganglionated plexi
}

\author{
Shun-ichiro Sakamoto, MD, Richard B. Schuessler, PhD, Anson M. Lee, MD, Abdulhameed Aziz, MD, \\ Shelly C. Lall, MD, and Ralph J. Damiano, Jr, MD
}

\begin{abstract}
Objective: Surgical ablation of ganglionated plexi has been proposed to increase efficacy of surgery for atrial fibrillation. This experimental canine study examined electrophysiologic attenuation and recovery of atrial vagal effects after ganglionated plexi ablation alone or with standard surgical lesion sets for atrial fibrillation.
\end{abstract}

\begin{abstract}
Methods: Dogs were divided into 3 groups: group $1(n=6)$ had focal ablation of the 4 major epicardial ganglionated plexi fat pads, group $2(n=6)$ had pulmonary vein isolation with ablation, and group $3(n=6)$ had posterior left atrial isolation with ablation. All fat pads were ablated. Sinus and atrioventricular interval changes during bilateral vagosympathetic trunk stimulation were examined before and both immediately and 4 weeks after ablation. Vagally induced effective refractory period changes and mean QRST area changes (index of local innervation) were examined in 5 atrial regions.
\end{abstract}

Results: Sinus and atrioventricular interval changes and heart rate variability decreased immediately after ablation, but only sinus interval changes were restored significantly after 4 weeks in all groups. Ablation-modified vagal effects on effective refractory period or QRST area changed heterogeneously in groups 1 and 2. In group 3 , regional vagal effects were attenuated extensively postablation in both atria. Posterior left atrial isolation with ablation incrementally denervated the atria. In the long term, vagal stimulation increased QRST area changes relative to control values in all groups. Heart rate variability was also assessed.

Conclusions: Ganglionated plexi ablation significantly reduced atrial vagal innervation. Restoration of vagal effects at 4 weeks suggests early atrial reinnervation. (J Thorac Cardiovasc Surg 2010;139:444-52)

Parasympathetic tone has a profound effect on atrial electrophysiology. It results in shortening of refractory periods without alteration in conduction. ${ }^{1,2}$ Combined with sympathetic tone, it facilitates triggered activity, which can be a factor in the induction of atrial fibrillation (AF). ${ }^{3}$ Parasympathetic and sympathetic fibers enter the heart along the pulmonary veins, superior vena cava (SVC), and aorta. ${ }^{4-6}$ They form synaptic junctions with other fibers in multiple ganglionated plexi (GP). These GP are part of a integrated network of nerves that form an intrinsic nervous system for the atria that modulates and controls the release of neural transmitters within the atria. There are $619 \pm 88 \mathrm{GP}$ in the canine atria; however, 4 fat pads have been found to contain large concentrations of GP. The 4 fat pads are located (1) lateral to

\footnotetext{
From the Division of Cardiothoracic Surgery, Department of Surgery, Washington University School of Medicine, Barnes-Jewish Hospital, St Louis, Mo.

Disclosures: Richard B. Schuessler reports lecture fees and grant support from Atricure; Ralph J. Damiano, Jr, reports consulting and lecture fees from Atricure and Medtronics, consulting fees from Medical CV, lecture fees from Edwards, and grant support from Atricure and Estech.

Supported by National Institutes of Health grants R01 HL 032257, R01 HL085113, and T32 HL007776.

Received for publication Feb 20, 2009; revisions received April 1, 2009; accepted for publication April 23, 2009; available ahead of print Sept 10, 2009.

Address for reprints: Richard B. Schuessler, PhD, Division of Cardiothoracic Surgery, 660 S Euclid, Campus Box 8234, Washington University School of Medicine, St Louis, MO 63110 (E-mail: schuesslerr@wustl.edu).

$0022-5223 / \$ 36.00$

Copyright (c) 2010 by The American Association for Thoracic Surgery doi:10.1016/j.jtcvs.2009.04.056
}

the right pulmonary vein (RPV), (2) below the inferior vena cava (IVC) on the left atrium (LA), (3) at the junction of the SVC and aorta, (4) and anterior to the superior left pulmonary vein (LPV; Figure 1) Stimulation of these fat pads slows heart rate and conduction in the atrioventricular node and decreases refractory period in the atrial myocardium. ${ }^{7}$ Stimulation of these fat pads in combination with sympathetic agonist can induce premature atrial beats and AF. Ablation of these fat pads has been shown to reduce the inducibility of $\mathrm{AF}^{8}$; however, selective ablation of only 1 of these fat pads can increase the vulnerability of the atria to AF by increasing the inhomogeneity of refractoriness. ${ }^{9}$

Clinical studies with catheter or surgical approaches suggest that in combination with a standard ablation approach, GP ablation increases the efficacy of the procedure. ${ }^{10-13}$ It has also been proposed to add GP ablation to surgical lesion sets to enhance results. ${ }^{13}$ Heart rate variability (HRV) data, however, suggest that reinnervation takes place after ablation of the GP. ${ }^{10}$ In a canine autotransplant model, vagal reinnervation has been demonstrated functionally and histologically. ${ }^{14}$ The data suggest that the innervation is remodeled inhomogeneously. Reinnervation has also been demonstrated after heart transplant in human patients. ${ }^{15-17}$ The acute and chronic effects on regional atrial innervation of GP ablation, alone or combined with standard ablation lesions sets, remain unclear. This study was designed to determine the acute and chronic electrophysiologic effects of GP ablation, GP 


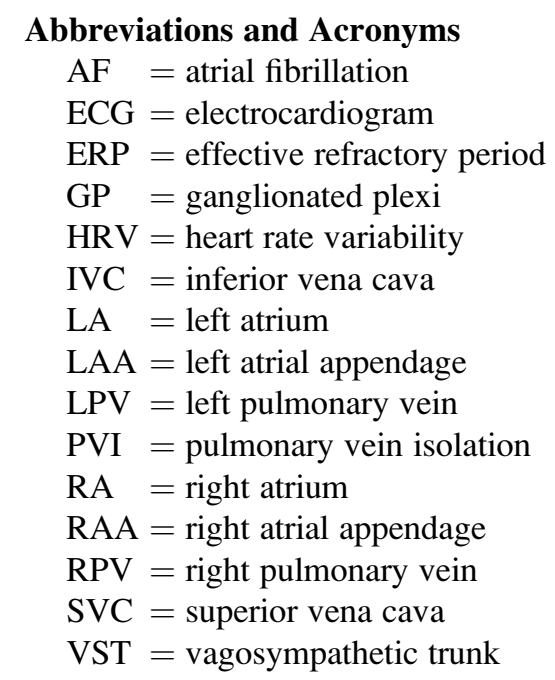

ablation plus isolation of the LPV and RPV (PVI), and GP ablation, PVI, and isolation of the intervening atria between the LPV and RPV (box lesion).

\section{MATERIALS AND METHODS \\ Surgical Preparation}

Eighteen adult mongrel dogs weighing between 25 and $30 \mathrm{~kg}$ were divided randomly into 3 groups. (1) Group $1(n=6)$ underwent focal ablation of 4 epicardial GP fat pads located between the medial SVC and the aortic root (SVC-aortic fat pad), at the junction of the right atrium (RA) and RPV (RPV fat pad), at the IVC-LA junction (IVC-LA fat pad), and anterior to the superior LPV (LPV fat pad). (2) Group $2(\mathrm{n}=6)$ underwent PVI combined with GP ablation. (3) Group $3(n=6)$ underwent PVI and isolation of the intervening tissue between the LPV and RPV veins (box lesion) combined with GP ablation (Figure 1).

All animals were anesthetized with intravenous propofol $(5-7 \mathrm{mg} / \mathrm{kg}$ ), intubated with a cuffed endotracheal tube, and mechanically ventilated. An adequate level of anesthesia was maintained with inhaled isoflurane $(1 \%-3 \%)$. A limb-lead electrocardiogram (ECG) was monitored. A femoral artery catheter was inserted to monitor systemic arterial pressure continuously. Arterial blood samples were drawn every 30 minutes to determine arterial $\mathrm{Po}_{2}$, acid-base balance, and electrolyte levels.

A median sternotomy was performed. A catheter was inserted into the SVC through the internal thoracic vein to monitor central venous pressure continuously. After the bilateral vagosympathetic trunks (VSTs) were dissected free, the pericardium was opened and 3 electrode templates containing 252 unipolar electrodes were sutured on the RA and LA. The electrode templates were approximated to the atria with suture passed through the tissue and template and then secured with a snare. This allowed the temporary removal and replacement of the templates in exactly the same locations.

All animals received humane care in compliance with the "Guide for the Care and Use of Laboratory Animals" (www.nap.edu/catalog/5140.html). The study protocol was approved by the Animal Studies Committee at Washington University School of Medicine.

\section{Electrophysiologic Data Collection}

Propranolol $(0.2 \mathrm{mg} / \mathrm{kg})$ was given before the collection of baseline electrophysiologic data. Bilateral VSTs were stimulated simultaneously with a pulse generator (Grass Instruments, Quincy, Mass) with a frequency of $10 \mathrm{~Hz}$, amplitude of $10 \mathrm{~V}$, and 5-ms pulse width duration. A bipolar pacing electrode was placed on the RA appendage (RAA)and LA appendage (LAA). The RA and LA were mapped with custom-made electrode templates containing 252 unipolar electrodes. The electrode templates were constructed from a form-fitting silicon elastomer (Specialty Silicone Fabricators, Paso Robles, Calif) that fit snugly on the entire atrial epicardium and contained 0.5-mm diameter silver electrodes (Pacific Wire \& Cable, Inc, Santa Ana, Calif). The interelectrode distance was $5 \mathrm{~mm}$. The RAA bipolar electrode was used to pace at a cycle length of $300 \mathrm{~ms}$, with a 2-ms duration at twice the pacing threshold. Atrial electrograms were recorded during (1) spontaneous normal sinus rhythm, (2) continuous pacing, (3) continuous pacing with VST stimulation, and (4) spontaneous normal sinus rhythm with VST stimulation. Data were acquired with a personal computer-based data acquisition and analysis system. The system included custom software capable of data acquisition, management, display, and analysis. Unipolar electrograms were recorded at a gain of 125 with a frequency response of 0.5 to $1000 \mathrm{~Hz}$. Each channel was digitized at $2000 \mathrm{~Hz}$ with 12-bit resolution.

Programmed stimulation was applied to each electrode pair of the template on the RAA, inferior RA, LAA, inferior LA, and posterior LA, with and without VST stimulation. An 8-beat drive train (S1) at a basic cycle length of $300 \mathrm{~ms}$ was followed by premature extra stimulus (S2) at a coupling interval of $200 \mathrm{~ms}$. The coupling interval of the premature stimulus was shortened by 5 -ms increments. The longest coupling interval of the premature beat that failed to capture the atrium was determined as the local effective refractory period (ERP). All data were collected acutely (before and after the surgical ablation) and chronically (4 weeks after the initial operation) for each animal.

\section{Fat Pad Stimulation}

After data collection, high-frequency stimulation (1000 ppm, duration $0.3 \mathrm{~ms}, 1-6 \mathrm{~V}$ ) was applied to each fat pad with a bipolar handheld electrode (Max 10; AtriCure, West Chester, Ohio) and pacer (PACE 203H; Oscar, Inc, Denver, Colo) ${ }^{18}$ Sinus rate was monitored during stimulation. A $10 \%$ reduction in heart rate was considered to indicate a vagal effect.

\section{Surgical Ablation}

In group 1, the 4 epicardial fat pads were surgically removed, and the atrial tissue beneath the fat pad was focally ablated with the bipolar electrode that was used to stimulate the fat pad to create a transmural lesion. Each focal ablation time was set at 15 seconds. In groups 2 and 3, the RPV fat pad was completely dissected at the interatrial groove. Blunt dissection was then used to allow an umbilical tape to be passed around each pulmonary vein. A bipolar clamp ablation device (AtriCure) was placed around the RPV and LPV with a cuff of surrounding atrial tissue and ablated. GP ablation was confirmed by repeated stimulation.

In group 3, a lesion was created between the superior RPV and LPV and then between the inferior RPV and LPV. These lesions were created by inserting the lower jaw of the clamp into the LA through a pursestring suture. After the ablations, the atrial tissue in the bilatera pulmonary veins and posterior wall of the LA was paced to ensure that the ablation had created a transmural lesion with conduction block. After completion of surgical ablation in each group, the electrode templates were reattached and electrophysiologic data were acquired. The chest was closed in layers, negative pressure was reestablished in the pleural cavity, and the animal was allowed to recover. Antibiotics were injected intramuscularly for 5 postoperative days, and analgesics were given as needed.

\section{Continuous ECG Recordings}

All animals underwent 24-hour ECG recordings, which were obtained preoperatively and at $1,7,14,21$, and 27 postoperative days with a 3 -channnel digital recorder (IQmark, Brentwood, Calif). HRV analysis was performed offline. The ECG signal was sampled at $200 \mathrm{~Hz}$. Premature beats, arrhythmias, electrical noise, and other aberrant ECG signals were excluded 
Group 1

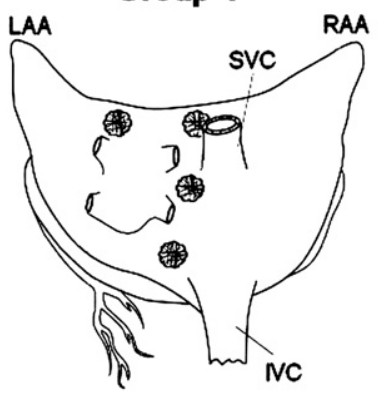

Group 2

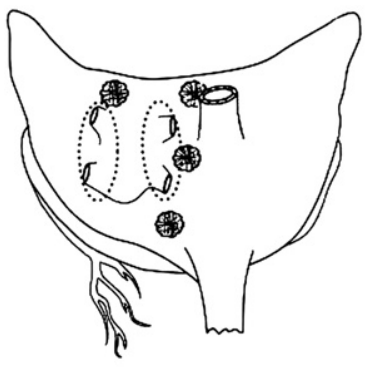

GP Fat Pad

Purse Suing
(2)

())

Radiofrequency Ablation ...

FIGURE 1. Surgical ablation sets in each group. $L A A$, Left atrial appendage; $S V C$, superior vena cava; $R A A$, right atrial appendage; $I V C$, inferior vena cava; $G P$, ganglionated plexi.

from the HRV analysis. Time-domain measures and power spectral analysis were performed. Time-domain HRV parameters were SD of normal R-R intervals, root mean square of SD, and triangular index. Frequency-domain HRV parameters were high-frequency $(0.16-0.4 \mathrm{~Hz})$ power and low-frequency $(0.04-0.15 \mathrm{~Hz})$ power.

\section{Data Analysis}

Spontaneous normal sinus intervals before and during vagal stimulation were measured. Atrioventricular intervals were calculated from data obtained during continuous pacing. Both interval changes were expressed as the percentage of lengthening (positive value) or shortening (negative value). ERP changes before and during vagal stimulation were expressed as percentages of baseline.

Atrial activation sequence data were analyzed with a computer program to determine the local activation times from unipolar tracings. The time of local activation was defined as the peak negative derivative of the major deflection of the unipolar complex. All electrograms were edited visually to verify accuracy of the computer-selected activation times. Computer-gener- ated activation sequence maps were constructed from the recordings. Activation maps were displayed on a 3-dimensional surface model of the canine atrium.

QRST area measurements of local electrograms have been used to detect changes in atrial electrical activity induced by stimulation of individual nerves. ${ }^{19}$ The change in area of QRST deflection in the unipolar waveform is a reflection of changes in action potential duration, which is sensitive to sympathetic and parasympathetic stimulation. QRST area was determined from an isoelectric diastolic baseline calculated just after the pacing spike. The area calculation started $10 \mathrm{~ms}$ after the local activation and proceeded for $50 \mathrm{~ms}$ with VST stimulation (Figure 2, A). The absolute value of the difference in the area under the curve between the paced and paced with stimulation data was calculated and normalized to the paced data. To determine the level of noise in the data (used as control value), 2 successive beats of paced data without stimulation were analyzed. Data from 252 sites were collected and divided into 5 anatomic regions consisting of the superior RA, inferior RA, superior LA, inferior LA, and posterior LA. The mean QRST area change in each atrial region was then calculated (Figure 2,B).
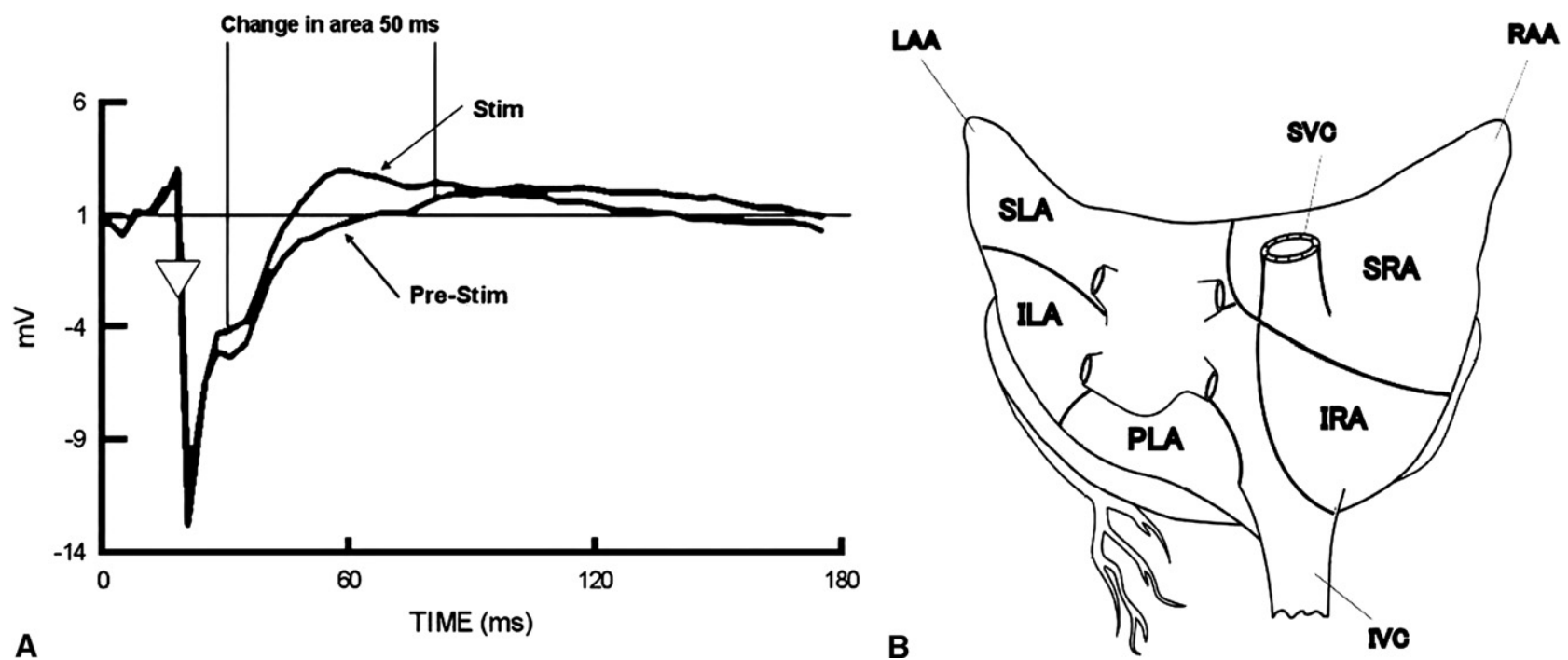

FIGURE 2. A, Calculation of change in QRST area for unipolar electrographic analysis. Stim, Stimulation; Pre-Stim, before stimulation. B, Five atrial regions in QRST analysis. RAA, Right atrial appendage; $L A A$, left atrial appendage; SVC, superior vena cava; $S L A$, superior left atrium; $S R A$, superior right atrium; ILA, inferior left atrium; PLA, posterior left atrium; IRA, inferior right atrium; $I V C$, inferior vena cava. 


\section{Statistical Analysis}

All continuous values were expressed as mean \pm SE. All data were tested for normality (Schapiro-Wilks test) and equality of variance (Bartlett test). If needed, a log transform was performed, and the data were retested for normality and equality of variances. Analysis of variance was used to test the null hypothesis. The data over time (preablation, postablation, 27 days after ablation) within a group were compared with the repeated analysis of covariance model with 1 factor. Post hoc multiple comparisons were made with a Fisher least significant difference test or contrasts with a Dunn-Sidak correction. All calculations were performed with the SPSS 11.5 statistical package (SPSS Inc, Chicago, Ill).

\section{RESULTS}

\section{Vagal GP Fat Pad Stimulation}

Heart rate slowing was observed during stimulation of $33 \%$ of fat pads (24/72). The RPV fat pad was the most consistent in reducing heart rate during stimulation. Heart rate slowed $77 \%$ of the time during RPV fat pad stimulation $(14 / 18)$ by an average of $26.8 \pm 3.0$ beats/min $(14 \%-$ $49 \%$ ). Spontaneous AF was observed during stimulation of the IVC-LA fat pad in 3 animals. Five animals $(27 \%)$ showed heart rate slowing during stimulation of the SVCaortic fat pad, and only 2 animals (11\%) showed slowing during stimulation of the superior LPV fat pad. Slowing of heart rate was eliminated after the fat pads were removed and the underlying atrial tissue was ablated.

\section{Sinus and Atrioventricular Interval Changes}

Postoperatively, ablation significantly decreased both sinus and atrioventricular interval changes during VST stimulation from control values in all groups (Figure 3). At 4 weeks, there was a partial restoration in heart rate during
VST stimulation. Sinus interval changes were greater that post ablation values in all groups (groups 1, 2, and 3, $8.5 \pm$ 1.7 vs $0.8 \pm 0.4, P=.009,4.9 \pm 1.6$ vs $-0.5 \pm 0.6, P=$ .02 , and $8.8 \pm 1.7$ vs $-0.2 \pm 0.6, P=.047$, respectively; Figure 3). At 4 weeks, there was a partial restoration in the change in heart rate during VST stimulation. There were no significant differences in sinus $(P=.60)$ and atrioventricular $(P=.12)$ interval changes with time between the groups.

\section{Heart Rate Variability}

Figure 4 shows the change in HRV (SD of normal R-R intervals, root mean square of $\mathrm{SD}$, triangular index, high-frequency power, and low-frequency power) in all groups. Each group was similar to the others in that the value of every parameter significantly decreased immediately after ablation $(P<.001)$ and remained low relative to preablation values $(P<.001)$. All measures of HRV increased from postoperative day 1 to postoperative day $27(P<.007)$. There were no significant differences in any parameter of HRV between groups $(p>.18)$.

\section{Sinus Activation Mapping During Vagal Stimulation}

During control normal sinus rhythm, the earliest site of activation was observed at the cranial site of the sinus node at the juncture of the SVC and RAA in most animals (17/18; Figure 5, A). Vagal stimulation produced a caudal shift of the earliest activation site in half of the animals in each group (Figure 5, B). Two animals demonstrated multicentric activation, with 2 separate origins of activation. After the surgical ablation, the caudal shift of the earliest activation no longer

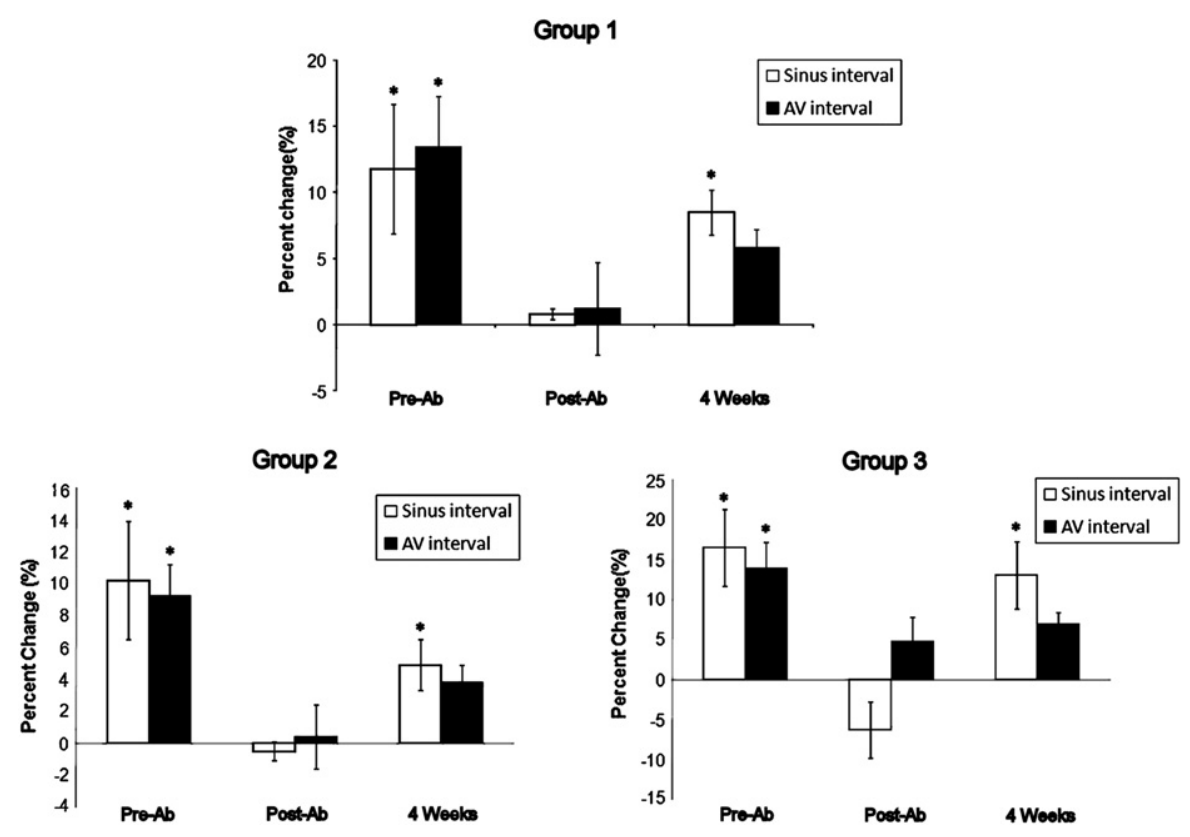

FIGURE 3. Change in sinus and atrioventricular $(A V)$ intervals in groups 1, 2, and 3. Pre-Ab, Before ablation; Post-Ab, immediately after ablation. Asterisk indicates $P<.05$ for control versus vagosympathetic trunk stimulation. 

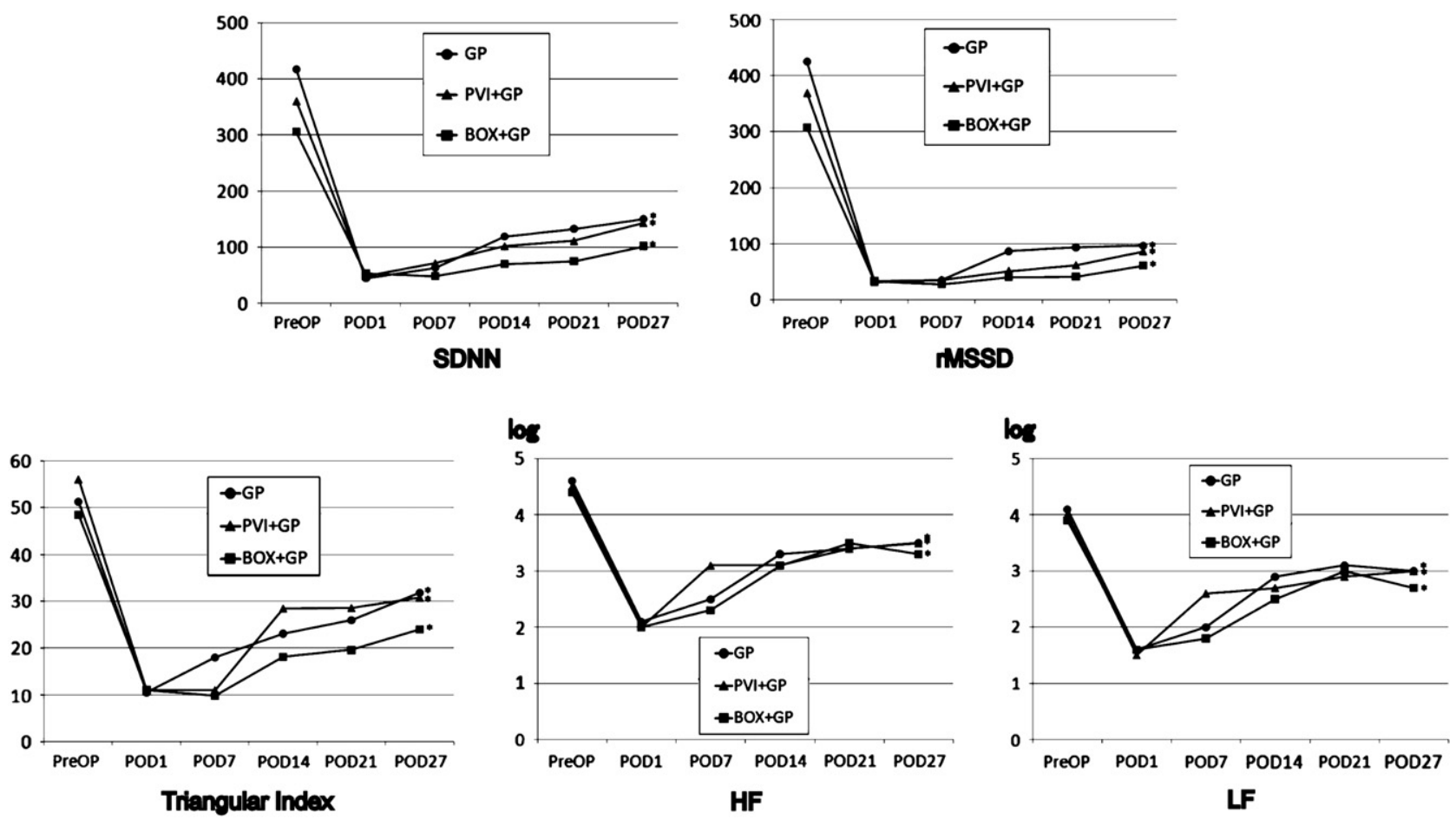

FIGURE 4. Changes in heart rate variability. $G P$, Ganglionated plexi ablation; $P V I$, pulmonary vein isolation; $B O X$, isolation of atria between right and left pulmonary veins; $S D N N$, SD of normal R-R intervals; $r M S S D$, root mean square of SD; $H F$, high-frequency power; $L F$, low-frequency power.

occurred in groups 1 and 2 (Figure 5, C). Two animals in group 3 demonstrated a cranial shift of origin with shortening of the sinus interval (sinus interval changes of $-2.8 \%$ and $-13 \%$ ). Four weeks after the ablation, there were 4 animals (1 in group 1, 1 in group 2, 2 in group 3) that demonstrated caudal or cranial shift with a prolonged sinus interval (sinus interval change of $6.1 \% \pm 1.3 \%$; Figure $5, E$ ).

\section{Mean QRST Area Change}

Figure 6 demonstrates the mean QRST area change in the 5 atrial regions in each group. A significant increase in the magnitude relative to the control (prestimulation value) is indicated by shading. Vagal stimulation led to QRST area changes greater than control values in all groups, except for the inferior RA in group 1. After ablation, group 1 showed no significant QRST area changes in the superior and inferior LA and showed increased area changes in the inferior RA. In groups 2 and 3, vagal effects were extensively attenuated even in the RA. Group 2 showed no QRST area changes in the superior and inferior RA and the superior and inferior LA. In group 3, area changes were absent in the inferior RA and the superior and posterior LA. Four weeks after ablation, QRST area changes during vagal stimulation were present in all groups and in all regions relative to the postablation values except the inferior LA in group $3(P<.05)$. In addition, vagal stimulation increased QRST area changes relative to preablation values long-term in 2 regions in groups 1 and 2 (Table 1).

\section{Change in ERP}

Figure 7 shows ERP changes during vagal stimulation before ablation, immediately after ablation, and at 4 weeks after ablation in each group. In group 1, ERP decreased in every region and remained low at 4 postoperative weeks; however, there were no significant changes in ERP in any atrial region immediately after ablation. The only significant change was an attenuated ERP change in the inferior RA at 4 weeks (preablation vs 4 weeks $5.8 \% \pm 2.0 \%$ vs $8.3 \% \pm 3.0 \%, P=.013$ ). In group 2 , ERP decreased in 3 atrial regions and increased in the RAA and LIA immediately after ablation. Significantly smaller ERP changes were observed in the RAA (preablation vs 4 weeks $9.1 \% \pm 3.5 \%$ vs $4.1 \% \pm 3.2 \%, P=.041)$ and LAA (preablation vs 4 weeks $12.5 \% \pm 3.3 \%$ vs $1.6 \%$ $\pm 1.0 \%, P=.021)$. In group 3, ablation decreased ERP in every atrial region. At 4 weeks, these changes remained significantly low relative to preablation values in all regions except the RAA $(P=.21)$.

\section{DISCUSSION}

The major finding of this study was that GP ablation, both alone and combined with standard surgical ablation sets for $\mathrm{AF}$, extensively reduced the effects of bilateral VST stimulation not only on the atrial conduction system but also on the atrial myocardium. Within 4 weeks, however, there was a return of parasympathetic effects on the sinus node and atrial myocardium. In addition, at 4 weeks VST 

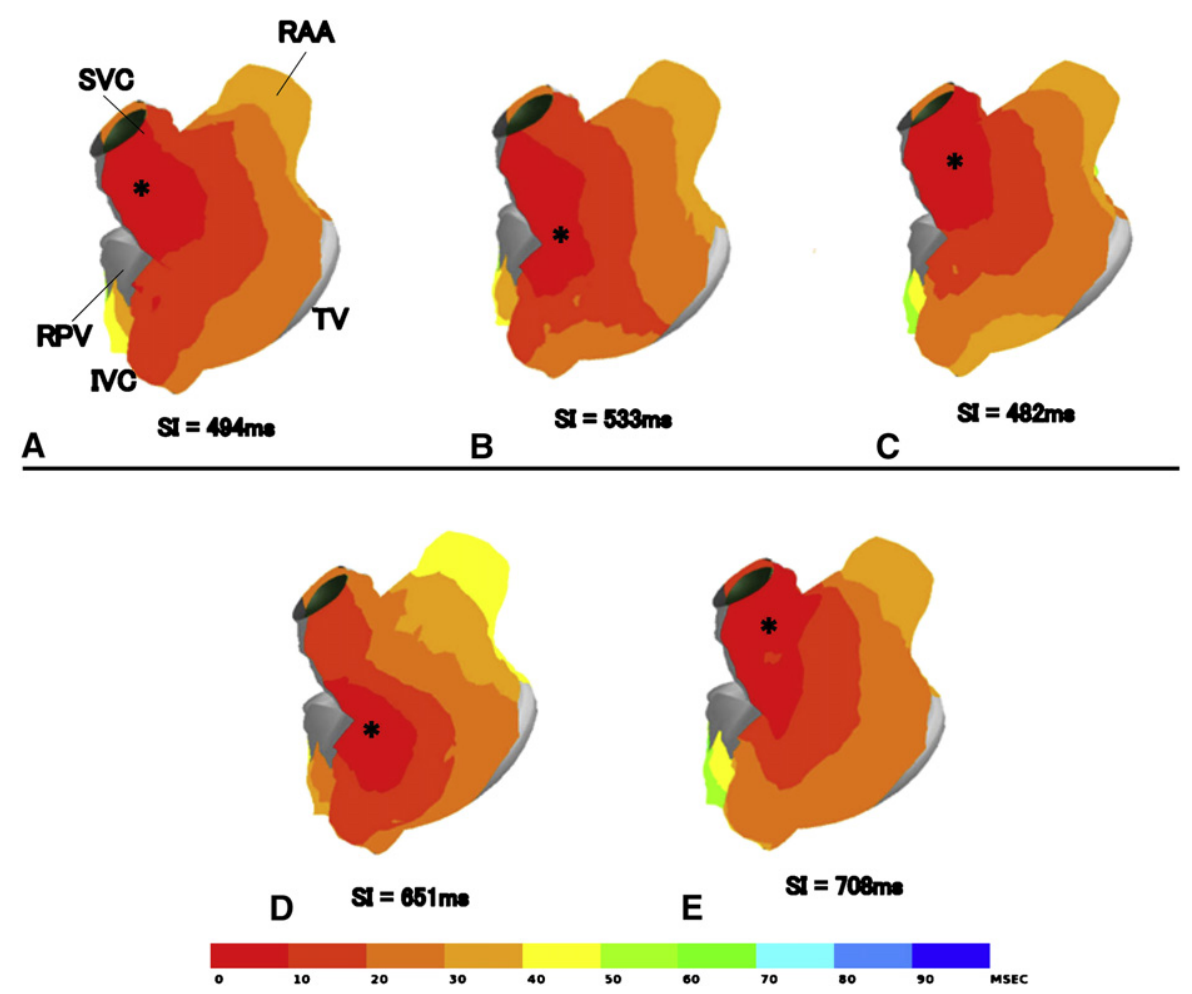

FIGURE 5. Upper panel represents atrial activation maps constructed from sinus rhythm without vagal stimulation (A), sinus rhythm with vagal stimulation before ablation (B), and sinus rhythm with vagal stimulation immediately after ablation (C). Lower panel represents sinus activation maps without vagal stimulation (D) and with vagal stimulation (E) at 4 weeks in same animal. Maps represent lateral view in right atrium. Asterisks indicate earliest activation site in right atrium. Atrial activation is shown with color coding in 10-ms increments. Numbers below figure indicate sinus interval times in each activation. RAA, Right atrial appendage; $S V C$, superior vena cava; $R P V$, right pulmonary vein; $T V$, tricuspid valve; $I V C$, inferior vena cava.

stimulation elicited a hyperresponse in some areas of the atria relative to the control value before ablation.

In all groups, the sinus and atrioventricular nodal responses to VST stimulation were almost completely eliminated immediately after ablation. HRV also suggested that the normal neural tone in the intact animal was absent. The fact that there were no differences between any of the ablation groups suggests that the 4 GPs ablated in this study modulated most of the entire functional neural input into the sinus and atrioventricular nodes. In a canine study by Lall and colleagues ${ }^{20}$ the lesions of the Cox maze IV or PVI without GP ablation only reduced parasympathetic and sympathetic effects in the sinus node and did not affect the atrioventricular node. This suggests that our addition of the GP ablations increased the extensiveness of the denervation acutely.

The early recovery in our study of neural function of the sinus node suggests that rapid parasympathetic reinnervation occurs in the dog. Recovery of the parasympathetic effects on the sinus node also were evident in the atrial activation mapping, which showed a caudal or cranial shift of the earliest activation site during VST stimulation. Even though the atrioventricular nodal response to VST stimulation was not significant at 4 weeks, there was a trend toward a return of innervation. The lack of a complete return of parasympathetic effects on the atrioventricular node suggest that the reinnervation was not uniform. At 4 weeks, the parasympathetic effects on the atrioventricular node were still attenuated, and it is unknown whether they would return to the preablation levels after a longer time.

The effects of VST stimulation on the regional atrial myocardium, as measured by changes in the local QRST area of the unipolar electrogram, suggest that none of ablation sets completely eliminated the regional parasympathetic effects on atrial myocardium. Before ablation, this technique was able to demonstrate a change in local repolarization in almost all the regions in all of the groups. The addition of the surgical lesion sets to GP ablation increased the area of atrial myocardium that lost the parasympathetic effect on repolarization during VST stimulation. At 4 weeks, however, the effects during VST stimulation had returned in all 3 groups in virtually every region. The effects of VST stimulation on repolarization were greater at 4 weeks than during the preablation control period in some of the regions, and the extent of this increase was dependent on the lesion set. Only 1 region showed the hyperresponse at 4 weeks in the GP ablation group (group 1), 2 regions in the PVI group (group 2) had a greater response, and all 5 regions in the box lesion 


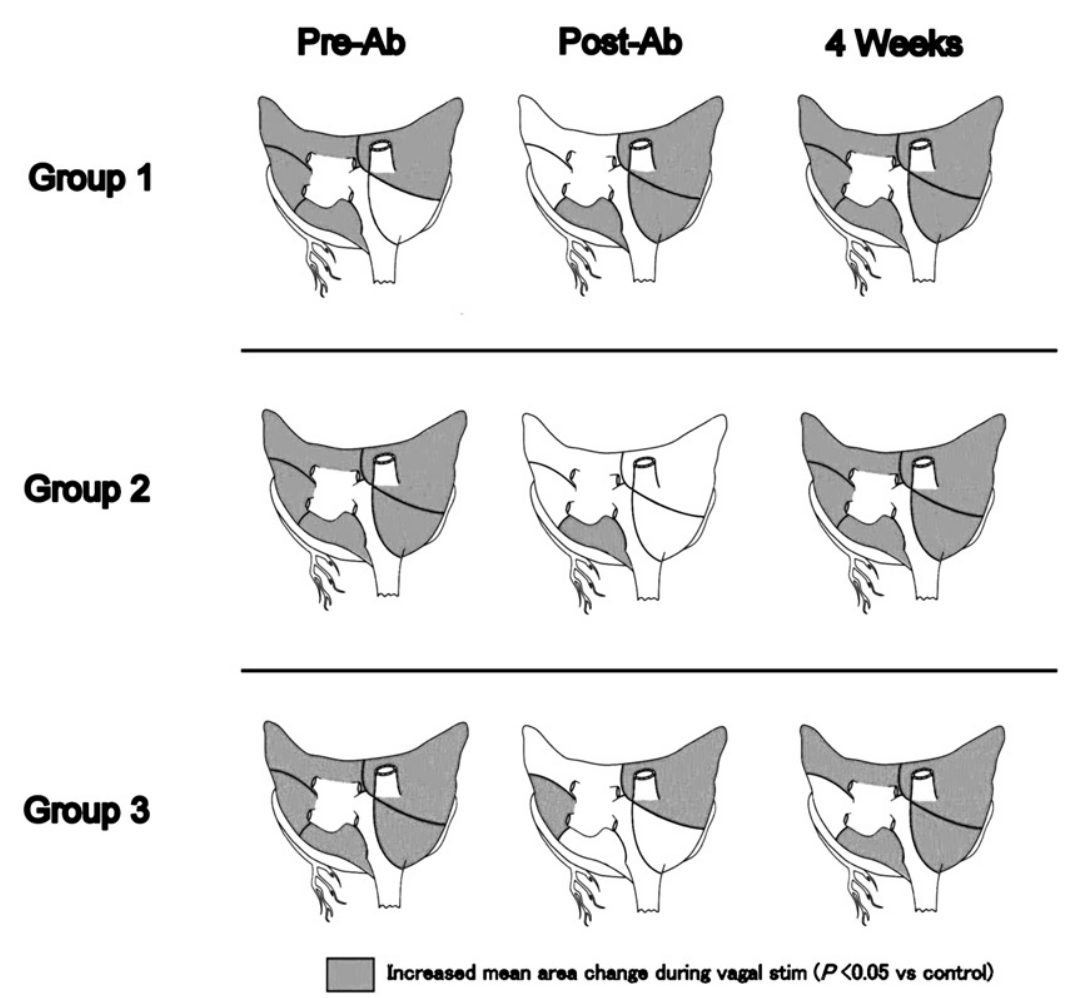

FIGURE 6. Mean QRST area changes at 5 atrial regions before ablation (Pre-Ab), immediately after ablation (Post-Ab), and 4 weeks after ablation. Significantly increased area changes against control values $(P<.05)$ are indicated by shading. stim, Stimulation.

group (group 3) responded with a greater change. These data suggest that the return of the parasympathetic innervation was not only rapid but dependent on the ablation set. A possible mechanism for the hyperresponse to vagal stimulation at 4 weeks is neural remodeling. Kaseda and coworkers ${ }^{21}$ showed that vagal denervation in the canine atrium causes a supersensitive response to acetylcholine in the long term, implying an increased number or density of muscarinic receptors. Sympathetic nerve sprouting and hyperinnervation have also been shown in the infarcted heart. ${ }^{22}$ There have been no previous reports, however, regarding hyperreinnervation of the parasympathetic nerves in the atria. The ERP data were more ambiguous, although they did suggest that ablation decreases the effects of VST stimulation. The variability in the data may have been due to the time needed to assess the ERP with the extra stimulus technique. The effects of VST stimulation may have been attenuated during this time.
The data in our study are similar to those of Oh and associates, ${ }^{23}$ who demonstrated that ablation of RPV and IVCLA fat pads eliminated the parasympathetic effects on the sinus and atrioventricular nodes and local refractory period acutely in the dog. They also showed, however, that sinus node, atrioventricular node, refractory periods, and inducibility of AF returned to preablation levels at 4 weeks. In dogs, reinnervation occurred rapidly in our study, similar to other studies. There is convincing evidence that this would also be the case in human beings. Heart transplant achieves total sympathetic and parasympathetic denervation. ${ }^{15,16}$ Sympathetic reinnervation is observed frequently after human heart transplant; however, parasympathetic reinnervation has been reported in only a few cases. Bernardi and coworkers ${ }^{17}$ observed that the occurrence of vagal reinnervation depends on the type of surgery. They confirmed the presence of parasympathetic reinnervation in recent heart

TABLE 1. QRST area change during vagal stimulation (4 weeks vs preablation)

\begin{tabular}{|c|c|c|c|c|c|c|c|c|c|}
\hline \multirow[b]{2}{*}{ Region } & \multicolumn{3}{|c|}{ Group 1} & \multicolumn{3}{|c|}{ Group 2} & \multicolumn{3}{|c|}{ Group 3} \\
\hline & Preablation & $4 \mathrm{wk}$ & $P$ value & Preablation & $4 \mathrm{wk}$ & $P$ value & Preablation & $4 \mathrm{wk}$ & $P$ value \\
\hline Superior RA & $33.2 \pm 4.2$ & $30.8 \pm 2.3$ & .13 & $26.6 \pm 2.1$ & $25.5 \pm 2.5$ & .99 & $27.7 \pm 1.7$ & $13.8 \pm 1.0$ & $<.001$ \\
\hline Inferior RA & $27.5 \pm 1.9$ & $37.9 \pm 3.7$ & .52 & $57.2 \pm 5.5$ & $70.2 \pm 6.2$ & $<.001$ & $39.4 \pm 2.3$ & $19.6 \pm 1.4$ & $<.001$ \\
\hline Superior LA & $26.8 \pm 1.8$ & $59.1 \pm 6.7$ & .005 & $43.5 \pm 7.2$ & $92.8 \pm 14.3$ & $<.001$ & $61.3 \pm 15.1$ & $19.7 \pm 1.8$ & $<.001$ \\
\hline Inferior LA & $36.4 \pm 4.3$ & $72.8 \pm 12.4$ & .69 & $51.9 \pm 7.5$ & $69.7 \pm 7.0$ & .08 & $48.7 \pm 6.9$ & $18.0 \pm 1.8$ & $<.001$ \\
\hline Posterior LA & $42.1 \pm 6.7$ & $77.0 \pm 8.4$ & .004 & $55.6 \pm 8.4$ & $60.4 \pm 6.6$ & .29 & $62.5 \pm 9.1$ & $32.3 \pm 7.5$ & $<.001$ \\
\hline
\end{tabular}

$R A$, Right atrium; $L A$, left atrium. 

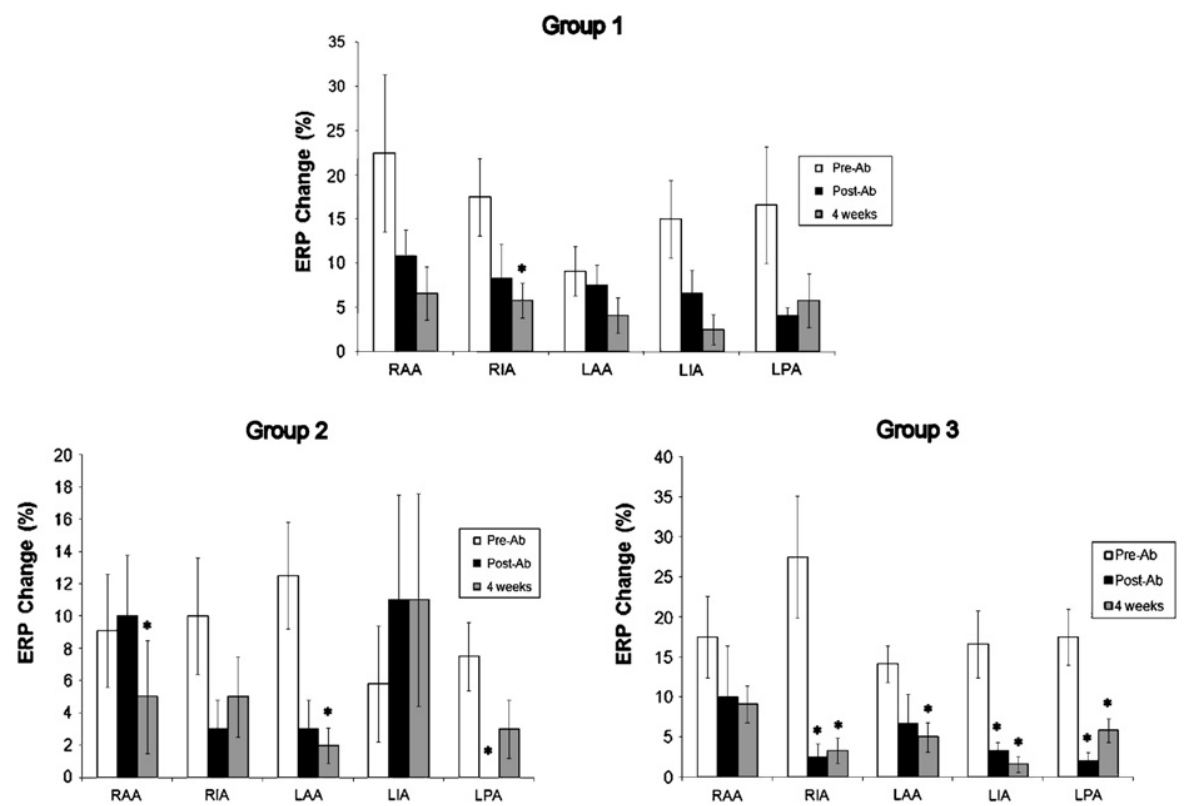

FIGURE 7. Changes in effective refractory period $(E R P)$ at 5 atrial regions in groups 1,2 , and 3 . Pre-Ab, Before ablation; Post-Ab, immediately after ablation; $R A A$, right atrial appendage; $R I A$, right inferior atrium; $L A A$, left atrial appendage; $L I A$, left inferior atrium; $L P A$, left posterior atrium. Asterisk indicates $P<.05$.

transplant recipients (bicaval technique), suggesting that interrupted parasympathetic fibers may regenerate similar to the regeneration observed with sympathetic fibers. In a canine heart transplant model, it has been demonstrated histologically and functionally that vagal reinnervation occurs across the suture lines. ${ }^{14}$ In patients who had undergone catheter PVI ablation and functional neural ablation, there was an attenuation in multiple HRV parameters, which returned to preablation levels after 6 months. ${ }^{10}$

Recently, there has been great interest in combining standard ablation lesion sets with GP ablation. Clinical studies have reported that GP ablation may increase the success rate for terminating and preventing $\mathrm{AF}$ in catheter ablation of AF. GP ablation effects have appeared to last for 3 to 12 months. ${ }^{10,12}$ Follow-up is needed, however, to determine whether reinnervation increases the late failure rate.

GPs are mainly epicardial structures, but efferent fibers are located both subepicardially, intramuscularly, and subendocardially. Many surgical ablation devices are unable to ablate atrial tissue transmurally through the fat pad. In this study, the fat pads were therefore surgically removed to ensure that ablations were transmural. The loss of a response to VST stimulation confirmed that the ablation was complete. Likewise, ablations applied to the endocardial surface during catheter ablation may be inadequate for ablating the nerve fibers that traverse the epicardium and fat pad.

The results of our study suggest a cautionary approach to the addition of GP ablation to accepted surgical lesion sets, such as the Cox maze procedure or PVI. This study convincingly demonstrated functional reinnervation within a short postoperative period. In patients with vagally mediated $\mathrm{AF}$, reinnervation may recreate the original substrate for
$\mathrm{AF}$, potentially causing recurrence. This is of particular concern if GP ablation alone is used as a treatment. A more important concern is that the reinnervation may be nonuniform, as was seen in our study. This could create a new substrate for $\mathrm{AF}$ that did not originally exist in the patient. A welldesigned, prospective, randomized, controlled trial with long-term follow-up will be required to determine whether GP ablation alone or in combination with another standard approach is efficacious in the treatment of AF.

\section{Study Limitations}

There are quantitative differences in the number of ganglia and in the distribution of nerves between dogs and human beings. ${ }^{5}$ The number of nerves is smaller in dogs than in human beings, but this is due in part to differences in atrial size. In the dog, about $70 \%$ of all intrinsic ganglia supply the sinus node, whereas in human beings, more than half of the ganglia supply the atrioventricular node. Despite these differences, the innervation in human beings and dogs is very similar. Caution should still be used in extrapolating these findings to the clinical situation. Although this study showed a return of parasympathetic effects with direct thoracic nerve stimulation, we did not examine whether the intracardiac sympathetic and parasympathetic network had returned to preablation conditions.

We would like to thank Diane Toeniskoetter and Naomi Still for their assistance in the animal studies.

\section{References}

1. Schuessler RB, Bromberg BI, Boineau JP. Effect of neurotransmitters on the activation sequence of the isolated atrium. Am J Physiol. 1990;258(6 Pt 2): H1632-41. 
2. Schuessler RB, Grayson TM, Bromberg BI, Cox JL, Boineau JP. Cholinergically mediated tachyarrhythmias induced by a single extrastimulus in the isolated canine right atrium. Circ Res. 1992;71:1254-67.

3. Tai CT, Chiou CW, Chen SA. Interaction between the autonomic nervous system and atrial tachyarrhythmias. J Cardiovasc Electrophysiol. 2002;13:83-7.

4. Pauza DH, Pauziene N, Pakeltyte G, Stropus R. Comparative quantitative study of the intrinsic cardiac ganglia and neurons in the rat, guinea pig, dog and human as revealed by histochemical staining for acetylcholinesterase. Ann Anat. 2002;184: 125-36.

5. Pauza DH, Skripka V, Pauziene N. Morphology of the intrinsic cardiac nervous system in the dog: a whole-mount study employing histochemical staining with acetylcholinesterase. Cells Tissues Organs. 2002;172:297-320.

6. Pauza DH, Skripka V, Pauziene N, Stropus R. Morphology, distribution, and variability of the epicardiac neural ganglionated subplexuses in the human heart. Anat Rec. 2000;259:353-82.

7. Hou Y, Scherlag BJ, Lin J, Zhang Y, Lu Z, Truong K, et al. Ganglionated plexi modulate extrinsic cardiac autonomic nerve input: effects on sinus rate, atrioventricular conduction, refractoriness, and inducibility of atrial fibrillation. J Am Coll Cardiol. 2007;50:61-8.

8. Schauerte P, Scherlag BJ, Pitha J, Scherlag MA, Reynolds D, Lazzara R, et al. Catheter ablation of cardiac autonomic nerves for prevention of vagal atrial fibrillation. Circulation. 2000;102:2774-80.

9. Hirose M, Leatmanoratn Z, Laurita KR, Carlson MD. Partial vagal denervation increases vulnerability to vagally induced atrial fibrillation. J Cardiovasc Electrophysiol. 2002;13:1272-9.

10. Pappone C, Santinelli V, Manguso F, Vicedomini G, Gugliotta F, Augello G, et al Pulmonary vein denervation enhances long-term benefit after circumferential ablation for paroxysmal atrial fibrillation. Circulation. 2004;109:327-34.

11. Saad EB, Saliba WI, Marrouche NF, Natale A. Pulmonary vein firing triggering atrial fibrillation after open heart surgery. J Cardiovasc Electrophysiol. 2002;13:1300-2.

12. Scanavacca M, Pisani CF, Hachul D, Lara S, Hardy C, Darrieux F, et al. Selective atrial vagal denervation guided by evoked vagal reflex to treat patients with paroxysmal atrial fibrillation. Circulation. 2006;114:876-85.
13. Mehall JR, Kohut RM Jr, Schneeberger EW, Taketani T, Merrill WH, Wolf RK. Intraoperative epicardial electrophysiologic mapping and isolation of autonomic ganglionic plexi. Ann Thorac Surg. 2007;83:538-41.

14. Murphy DA, Thompson GW, Ardell JL, McCraty R, Stevenson RS, Sangalang VE, et al. The heart reinnervates after transplantation. Ann Thorac Surg. 2000;69:1769-81.

15. Wilson RF, Christensen BV, Olivari MT, Simon A, White CW, Laxson DD. Evidence for structural sympathetic reinnervation after orthotopic cardiac transplantation in humans. Circulation. 1991;83:1210-20.

16. Kaye DM, Esler M, Kingwell B, McPherson G, Esmore D, Jennings G. Functional and neurochemical evidence for partial cardiac sympathetic reinnervation after cardiac transplantation in humans. Circulation. 1993;88:1110-8.

17. Bernardi L, Valenti C, Wdowczyck-Szulc J, Frey AW, Rinaldi M, Spadacini G, et al. Influence of type of surgery on the occurrence of parasympathetic reinnervation after cardiac transplantation. Circulation. 1998;97:1368-74.

18. Sakamoto S, Voeller RK, Melby SJ, Lall SC, Chang NL, Schuessler RB, et al. Surgical ablation for atrial fibrillation: the efficacy of a novel bipolar pen device in the cardioplegically arrested and beating heart. $J$ Thorac Cardiovasc Surg. 2008;136:1295-301.

19. Page PL, Dandan N, Savard P, Nadeau R, Armour JA, Cardinal R. Regional distribution of atrial electrical changes induced by stimulation of extracardiac and intracardiac neural elements. J Thorac Cardiovasc Surg. 1995; 109:377-88.

20. Lall SC, Foyil KV, Sakamoto S, Voeller RK, Boineau JP, Damiano RJ Jr, et al. Pulmonary vein isolation and the Cox maze procedure only partially denervate the atrium. J Thorac Cardiovasc Surg. 2008;135:894-900.

21. Kaseda S, Zipes DP. Supersensitivity to acetylcholine of canine sinus and AV nodes after parasympathetic denervation. Am J Physiol. 1988;255(3 Pt 2):H534-9.

22. Zhou S, Chen LS, Miyauchi Y, Miyauchi M, Kar S, Kangavari S, et al. Mechanisms of cardiac nerve sprouting after myocardial infarction in dogs. Circ Res. 2004;95:76-83.

23. Oh S, Zhang Y, Bibevski S, Marrouche NF, Natale A, Mazgalev TN. Vagal denervation and atrial fibrillation inducibility: epicardial fat pad ablation does not have long-term effects. Heart Rhythm. 2006;3:701-8. 